\title{
Influence of scheduled restricted feeding on reentrainment of motor activity rhythm after a 6-h light-dark advance in rats
}

\author{
Breno T.S. Carneiro and John F. Araujo \\ Universidade Federal do Rio Grande do Norte, Natal, RN, Brazil
}

\begin{abstract}
Circadian rhythms are entrained to cyclic environmental cues such as the light-dark cycle and food availability. The suprachiasmatic nucleus of the hypothalamus is the main circadian oscillator in mammals and is entrained primarily by the lightdark cycle, although in some experimental situations food availability can affect its oscillation. Here we tested whether scheduled restricted feeding is capable of influencing the reentrainment of the motor activity rhythm (i.e., a behavioral rhythm controlled by the suprachiasmatic nucleus) to a 6-h phase advance of the light-dark cycle. Rats were housed in a standard $12 \mathrm{~h}: 12 \mathrm{~h}$ light-dark cycle for 18 days, and then the time of lights-off was advanced. Rats subjected to scheduled restricted feeding starting on the first day of the light-dark shift showed faster reentrainment of the motor activity rhythm compared with control rats maintained with ad libitum food. The results showed that scheduled feeding effectively accelerates reentrainment of a behavioral circadian rhythm and suggest that the procedure may affect activity in the suprachiasmatic nucleus during the stage of reentrainment to light-dark shifts. Keywords: circadian rhythm, reentrainment, scheduled restricted feeding, light-dark shift.
\end{abstract}

Received 22 September 2011; received in revised form 2 December 2011; accepted 4 December 2011. Available online 29 December 2011

\section{Introduction}

Circadian rhythms are present in different species and expressed as daily fluctuations in physiological, behavioral, and cognitive processes (Kondo et al., 1993; Campos, Silva, Fernandes, Araujo, \& Menezes, 2001; Simonetta, Migliori, Romanowski, \& Golombek, 2009). In mammals, these rhythms are entrained primarily by the 24-h daily light-dark (LD) cycle. Behavioral and physiological daily rhythms are orchestrated by the central circadian pacemaker located in the suprachiasmatic nucleus (SCN) of the hypothalamus (Dibner, Schibler, \& Albrecht, 2010), which receives direct LD information from the retina (Johnson, Morin, \& Moore, 1988; Costa, Santee, Cavalcante, Moraes, Santos, \& Britto, 1999; Nascimento et al., 2010).

Circadian rhythms, however, are not only entrained by LD cycles. Daily feeding opportunities have been shown to influence numerous physiological and

Breno T.S. Carneiro and John F. Araujo, Department of Physiology, Biosciences Center, Federal University of Rio Grande do Norte, Natal, RN, Brazil. Correspondence regarding this article should be directed to: Breno T.S. Carneiro, Universidade Federal do Rio Grande do Norte, Departamento de Fisiologia, Centro de Biociências, Campus Universitário, Av. Sen. Salgado Filho, s/n, Lagoa Nova, P.O. Box 1506, Natal, RN 59078-970, Brazil. Phone: +55 (84) 3215 3409, ext. 218. Fax: +55 (84) 3211 9206. E-mail: brenotercio@yahoo.com.br behavioral rhythms in mammals and other species (Mistlberger, 1994). The main characteristic of behavioral rhythms entrained by food is their anticipatory aspect. For example, when rats and mice are subjected to scheduled restricted feeding in which food is available at the same time daily for only a few hours (e.g., $2 \mathrm{~h}$ ), the animals anticipate the feeding time by increasing their locomotion 2-3 $\mathrm{h}$ before food availability (Mistlberger, 1994), a phenomenon referred to as "food-anticipatory activity" (FAA). The properties of FAA identified in past decades strongly indicate that this phenomenon is the manifestation of a food-entrainable circadian oscillator (Mistlberger, 2009) distinct from oscillators located in the SCN (Stephan, Swann, \& Sisk, 1979; Marchant \& Mistlberger, 1997). Despite the recognition that the integrity of the SCN is not necessary for the emergence of FAA, evidence has indicated that a specific type of feeding regimen can exert an effect on SCN-controlled activity rhythm and SCN oscillatory operation. For example, hypocaloric feeding $(50-75 \%$ of ad libitum ingestion) has been shown to promote the entrainment of activity rhythms in rats exposed to constant darkness (Caldelas et al., 2005) and enhance phase-shift responses to light pulses in mice (Mendoza, Graff, Dardente, Pevet, \& Challet, 2005).

Nocturnal rodents maintained under prolonged exposure to constant light become behaviorally arrhythmic (Daan \& Pittendrigh, 1976) as a result 
of the uncoupling of SCN neurons (Ohta, Yamazaki, \& McMahon, 2005). Interestingly, behavioral and SCN rhythmicity is restored by scheduled restricted feeding under constant light conditions (Mistlberger, 1993; Lamont, Diaz, Barry-Shaw, Stewart, \& Amir, 2005). Lamont et al. (2005) suggested that the SCN can become sensitive to feeding schedules depending on the state of its oscillatory function (i.e., the degree of coupling among oscillators). Another experimental procedure that produces uncoupling within the SCN is LD phase shifts (i.e., either delays or advances). In the laboratory, rats subjected to LD phase shifts take several days to reentrain their activity rhythms to the new LD cycle, accompanied by uncoupling between the ventral and dorsal regions of the SCN (Nagano et al., 2003). Humans can experience LD shifts when they travel through time zones, from west to east or viceversa, leading to symptoms of jet lag (Waterhouse, Reilly, \& Atkinson, 1997).

Given the results that have shown that uncoupling in the SCN occurs with LD shifts and that feeding schedules can alter SCN coupling and its responses to light, we assessed whether scheduled restricted feeding is capable of altering the speed of reentrainment of motor activity rhythm (i.e., SCN-controlled behavioral rhythm) after a 6-h phase advance of the LD cycle.

\section{Methods}

\section{Subjects}

Eighteen Wistar rats ( 8 males and 10 females, 3 - to 6-months of age) from our colony were used. Animals were divided into three groups: Ad lib $(n=6)$, restricted feeding (RF; $n=7$ ), and food deprivation (FD; $n=5$ ). Rats were housed individually in standard polypropylene cages $(40 \times 32 \times 17 \mathrm{~cm})$ placed in light-tight, ventilated wooden cabinets $(180 \times 55 \times 50 \mathrm{~cm})$ with timer control of the lighting conditions and controlled temperature $\left(23 \pm 1^{\circ} \mathrm{C}\right)$. The study was performed in compliance with the ethical principles of the Brazilian Society of Sciences of Laboratory Animals (SBCAL).

\section{Apparatus}

General motor activity was continuously recorded in 5-min intervals using Aschoff software (developed in our laboratory) and infrared motion sensors (Focus 2000, Aspex) positioned $15 \mathrm{~cm}$ above the cage lids. The graphical output of activity rhythm (i.e., actograms) and rhythm analyses were performed using El Temps software (A. Díez-Noguera, Universitat de Barcelona, 1999).

\section{Procedure}

The Ad lib and RF groups were under a $12 \mathrm{~h}: 12 \mathrm{~h}$ LD cycle with ad libitum access to food for 18 days. Baseline daily food intake was measured in the RF group on days 4 to 7 in which food was weighed on days 4 and 7 , and the average daily intake was calculated. On day 18 , food was removed from the RF group at ZT 11. By convention, ZT 12 denotes the onset of the dark phase. On day 19, the LD cycle was shifted by advancing the dark phase by $6 \mathrm{~h}$, and the restricted feeding schedule began for the RF group, with food provided manually for $2 \mathrm{~h}$ between ZT 17 and ZT 19 of the new LD cycle. Animals in the Ad lib group had their cabinets open and food cup manually disturbed for $10 \mathrm{~s}$ at the same ZTs. Food intake during restricted feeding was measured daily in the RF group. An additional control group (FD) was maintained with ad libitum food for 14 days under a 12 h:12 h LD cycle. On day 14, food was removed at ZT 11. On day 15, lights were not turned on, and food was returned at ZT 11 of the previous LD cycle, corresponding to ZT 17 of the new LD cycle in the Ad lib and RF groups. Animals were under constant darkness (DD) for the next 11 days. This group was used to determine whether absence or presence of food itself, created on the first day of the restricted feeding procedure in the RF group, phase-advances the motor activity rhythm.

\section{Statistical analysis}

Acrophases were determined by the serial analysis tool in El Temps for the 7 days before the LD shift and each day after the LD advance or DD. Differences, expressed in hours, were calculated between the acrophase on the days after the LD shift or DD and the mean of the 7 days before the lighting conditions changed, not including the day prior to the lighting change. Food intake in the RF group on restricted feeding days after the LD shift was calculated as a percentage of baseline daily food intake. Additionally, FAA (defined as the total activity in the $3 \mathrm{~h}$ preceding food availability) was calculated for the first 4 days of the LD shift as a percentage of total daily activity.

Phase advances in acrophase, the number of days needed for a complete 6-h phase advance, and FAA were compared between Ad lib and RF groups using $t$-tests for independent samples. All data are expressed as mean \pm SEM. Values of $p<.05$ were considered statistically significant.

\section{Results}

The dynamics of reentrainment after the LD shift were different between Ad lib and RF groups. Rapid reentrainment of the motor activity rhythm was clear in the RF group compared with Ad lib group (Figures $1 \mathrm{~A}$ and $1 \mathrm{~B})$. The FD group showed an entrained rhythm to the LD cycle and a free running rhythm in DD (i.e., when the endogenous period is expressed; Figure 1C). The phase advance of rhythm acrophase 
was larger in the RF group primarily during the first days after the LD shift (Figure 2A). Additionally, the group of animals fed ad libitum needed almost twice as many days to reentrain to the new LD cycle compared with the group that was subjected to scheduled restricted feeding $(7.83 \pm 1.24$ and $4.42 \pm$ .90 for ad lib and RF group, respectively; Figure 2B). In the FD group, we observed a small phase advance in acrophase on the first day of DD when food was returned. Nonetheless, increasing phase delays were observed during the subsequent days, indicating a free-running rhythm that drifted to the right and a period $>24$ h (Figure 2C; see also Figure 1C). Food intake in the RF group increased after the LD shift and stabilized between $50 \%$ and $60 \%$ of ad libitum conditions (Figure 3). Food-anticipatory activity during the first 4 days of the LD shift is shown in Figure 4, differing between groups only on the fourth day of food restriction. The development of FAA is thought to require 3 to 14 days (Mistlberger, 1994). Figure 4 shows that FAA in the present study began to develop on the fourth day of restricted feeding.

\section{Discussion}

The present study showed that scheduled restricted feeding is capable of modifying the dynamics of the reentrainment of the motor activity rhythm in response
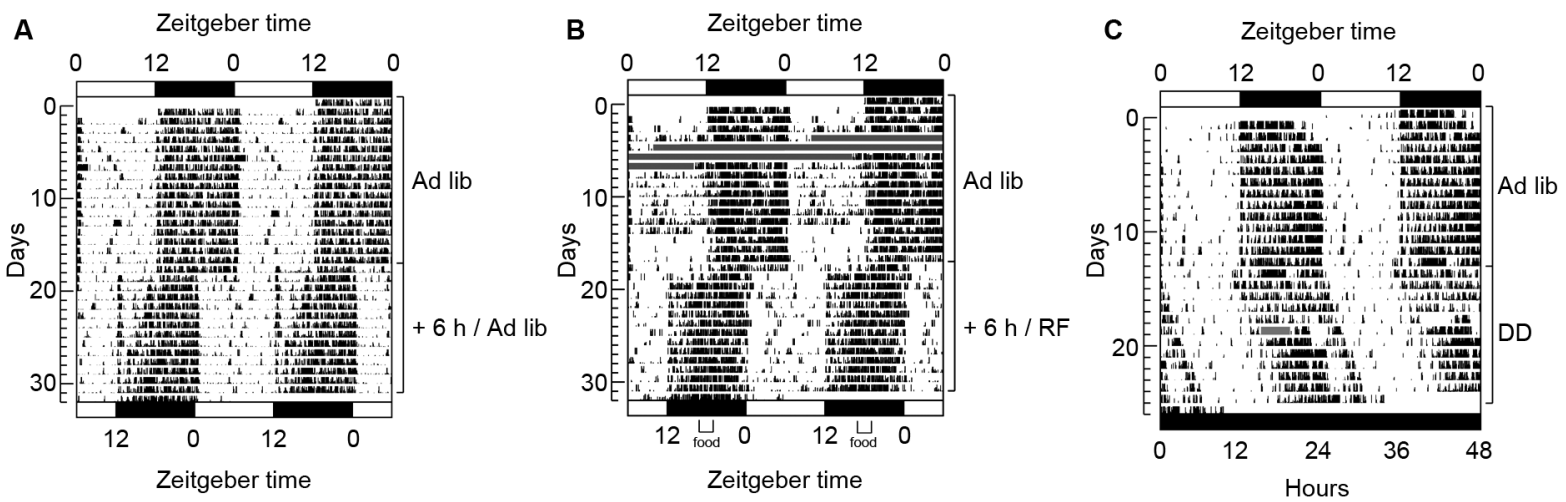

Figure 1. Double-plotted actograms of animals from the (A) Ad lib, (B) RF, and (C) FD groups. In this type of graphic representation, subsequent days are plotted from the top to bottom. "Double-plotted" means that day 1 is followed by day 2 on the same line. Day 2 will be repeated in the line below so that the line will contain days 2 and 3 and so on. On day 19, the LD cycle was advanced by $6 \mathrm{~h}$ in the Ad lib and RF groups. Starting on the first day of the new LD cycle (day 19), the RF group (B) received food only for $2 \mathrm{~h}$ in the middle of the dark phase, whereas the control group (A) had food ad libitum. The FD group (C) was food-deprived for $24 \mathrm{~h}$ starting at ZT 11 on day 14. On day 15, lights were not turned on and food was returned to the animals. The animals were in constant darkness for the next 11 days. A drift to the right is clear in the DD condition, showing that the rhythm was free running. Missing data are shown as horizontal gray bars.

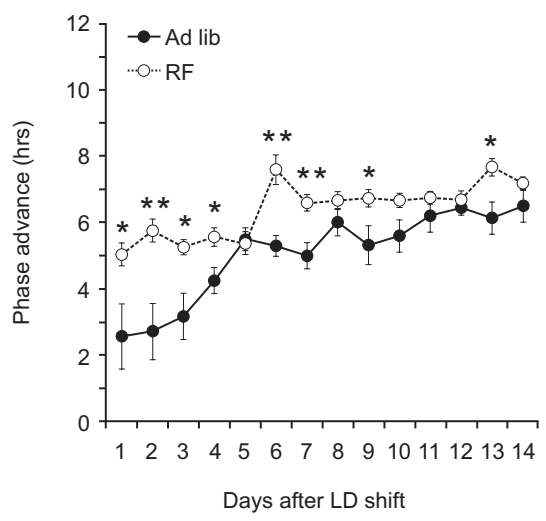

B

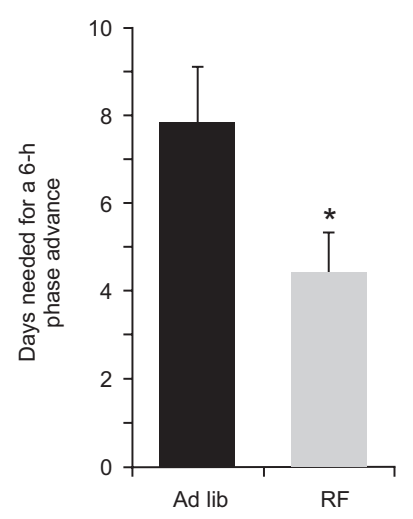

C

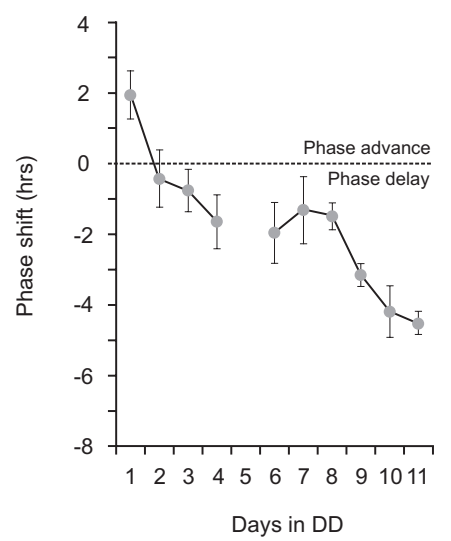

Figure 2. Phase shifts after a change in lighting condition. (A) Phase advance in acrophase in the RF and Ad lib groups. Advances were larger in the RF group essentially on the first days of the LD shift. (B) Number of days needed for a complete $6 \mathrm{~h}$ phase advance of acrophase. The Ad lib group took $7.83 \pm 1.24$ days to exhibit a $6 \mathrm{~h}$ phase advance, whereas the RF group took $4.42 \pm$ 0.90 days. (C) Phase shift of acrophase relative to previous LD cycle in the FD group. A small phase advance was observed on the first day of DD. The increasing delays indicate that the rhythm was free-running with a period longer than $24 \mathrm{~h}$ (see also Figure 1C). Due to missing data, acrophase was not obtained on day $5 .{ }^{*} p<.05, * * p<.01$ ( $t$-test for independent samples). 


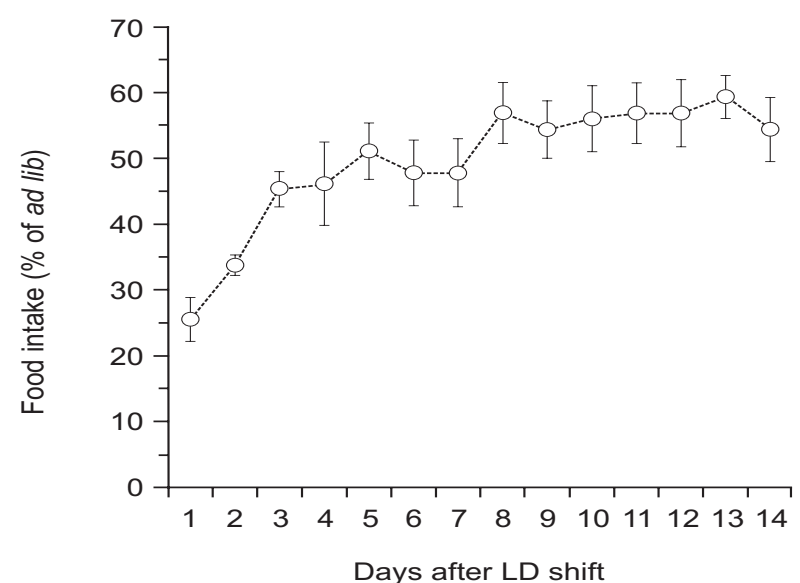

Figure 3. Food intake after LD shift. As expected, ingestion increased during the days of restricted feeding. Food intake stabilized at $\sim 50 \%$ and $60 \%$ of ad libitum ingestion.

to an advance of the LD cycle. Rapid reentrainment of the motor activity rhythm was observed in the RF group (Figures 1 and 2), indicating that the combination of an LD advance with scheduled restricted feeding may differentially affect the activity rhythm.

A possible mechanism involved in the rapid reentrainment of the RF group is masking. One could argue that acrophase advances were actually a false result attributable to increased FAA. Food-anticipatory activity would have led to a masking effect on activity and acrophase, shifting it in the direction of the new LD cycle. A masking effect would then be the cause of rapid reentrainment in the RF group. However, we saw that FAA was present only on day 4 of scheduled restricted feeding and the LD shift. Because we did observe large phase shifts on the first 3 days of the LD advance, we suggest that the phase advance of the rhythm was primarily attributable to an entrainment process. However, we cannot completely exclude the presence of a masking effect during reentrainment.

The activity circadian rhythm is dependent on the SCN in mammals because lesions abolish its expression (Stephan \& Zucker, 1972). However, the activity rhythm is also dependent on the food-entrainable oscillator (FEO) because it is restored in SCN-lesioned rodents subjected to restricted feeding schedules (Stephan, Swann \& Sisk, 1979; Marchant \& Mistlberger, 1997). The rapid reentrainment observed in the RF group may be attributable to an effect of scheduled restricted feeding directly on FEO activity. We previously proposed a theoretical model to explain how the FEO is organized and entrained (Carneiro \& Araujo, 2009). In this model we argue that the FEO is composed of several brain areas that are entrained by fluctuating humoral signals modified by food availability. These signals were likely

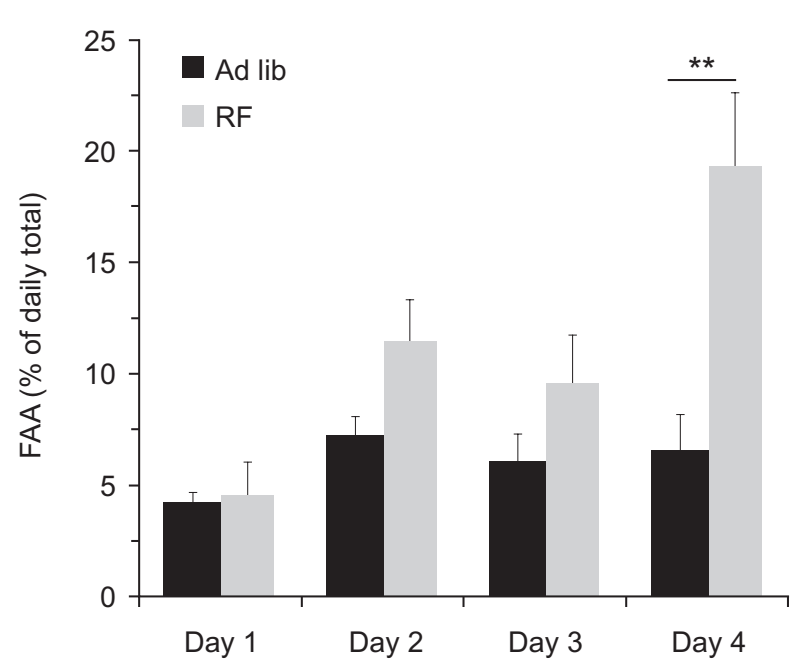

Figure 4. Food-anticipatory activity from day 1 to day 4 of restricted feeding/LD shift. Food-anticipatory activity is expressed as a percentage of total motor activity on each day (see Methods). A significant difference was observed only on the fourth day. ${ }^{* *} p<.01$ ( $t$-test for independent samples).

phase-shifted when food was restricted to the middle of the new dark phase in the present study. The rhythm shift may then be a consequence of FEO activation by humoral signals.

Under conditions of stable entrainment to LD cycles, SCN oscillation has been shown to not be affected by scheduled restricted feeding (Hara et al., 2001; Stokkan, Yamazaki, Tei, Sakaki, \& Menaker, 2001). However, Lamont et al. (2005) showed that when rats are arrhythmic because of prolonged exposure to constant light (i.e., a condition that produces the uncoupling of SCN neurons; Ohta et al., 2005), restricted feeding is capable of inducing FAA and restoring rhythmic oscillation within the SCN. Moreover, uncoupling within the SCN also occurs during reentrainment of circadian rhythms in response to LD advances or delays, specifically between ventral and dorsal regions (Nagano et al., 2003). A reduction of caloric intake by presentation of scheduled hypocaloric feeding has been reported to entrain activity rhythm in rats (Caldelas et al., 2005), alter SCN oscillations, and enhance phase shift responses to light stimulation in mice (Mendoza et al., 2005). Food intake in the RF group in the present study was $25-45 \%$ of ad libitum baseline on the first 4 days of the LD shift and stabilized between 50\% and $60 \%$ on subsequent days. Because caloric intake in the RF group was lower than baseline, feeding after the LD shift can also be considered hypocaloric. Therefore, animals subjected to RF in the present study ate a hypocaloric diet during an experimental procedure known to produce uncoupling within the SCN.

Any manipulation or signal capable of exerting an effect on SCN activity (Mrosovsky \& Salmon, 1987; Agostino, Plano, \& Golombek, 2007) or theoretically enhancing coupling among its oscillators can accelerate 
reentrainment of circadian rhythms in response to LD shifts. LD shift may render the SCN sensitive to cues associated with scheduled feeding, whereas hypocaloric feeding may enhance SCN responses to light during reentrainment. The combination of these events may explain the faster reentrainment observed in the RF group. Absence or presence of food itself on the food deprivation day and first day of the LD shift can also be argued to have an effect that phase-advances the SCN rhythm, thus leading to faster reentrainment of the activity rhythm. Nonetheless, Coleman \& Francis (1991) showed that 3 days of food deprivation did not phaseshift locomotor activity rhythm in rats under constant darkness but did when animals were under constant light. These phase shifts were correlated with activity levels on the last day of food deprivation. Indeed, food deprivation-induced phase shifts of the activity rhythm in hamsters did not emerge if locomotion was prevented by locking the running wheels (Mistlberger, Sinclair, Marchant, \& Neil, 1997). Phase shifts induced by food deprivation may be attributable to locomotion generated by the procedure and not by food deprivation or refeeding alone. In the present study we observed a small phase advance in acrophase on the first day of constant darkness when food was returned to the animals (see Figure 2C). However, the rhythm on subsequent days was not phase-advanced by food deprivation (see Figure $2 \mathrm{C}$ ). This is also visually apparent by the activity offset, which ran free according to the previous LD cycle (see Figure 1C). These results suggest that the phase of the circadian clock is little or unaffected by food removal or the reinstatement procedure.

Scheduled restricted feeding is associated with changes in peripheral humoral signals, many of which have been shown to act in brain regions and regulate food intake (Carneiro \& Araujo 2009). Some of these signals such as insulin, ghrelin, and leptin are known to bind receptors in the arcuate nucleus of the hypothalamus (ARC), an important area that integrates circulating signals to regulate food intake (Simpson, Martin, \& Bloom, 2009). Yi, van der Vliet, Dai, Yin, $\mathrm{Ru}$, \& Buijs (2006) reported that the ARC reciprocally communicates with the SCN. These investigators suggested that the ARC may serve as a pathway to communicate peripheral information to the SCN and modulate its activity. Through this connection, signals associated with restricted feeding may have an indirect effect on SCN activity, phase-shifting its oscillation and leading to rapid reentrainment after an LD advance. Notably, however, a direct effect of peripheral signals on the SCN is also possible. Leptin and ghrelin receptors are expressed in the SCN (Guan, Hess, Yu, Hey, \& van der Ploeg, 1997; Zigman, Jones, Lee, Saper, \& Elmquist, 2006). Additionally, SCN activity is phaseshifted by ghrelin and leptin application in vitro (Prosser \& Bergeron, 2003; Yannielli, Molyneux, Harrington, \&
Golombek, 2007). Therefore, a possible direct effect of these factors should be taken into consideration in the process of the reentrainment of activity rhythms to new LD cycles in rats under scheduled restricted feeding.

Another possible explanation for our results is that the two processes mentioned above occur simultaneously. Scheduled restricted feeding or its associated signals may affect FEO and SCN activity at the same time, generating a combined response of these two circadian oscillators that leads to rapid entrainment of the activity rhythm. Indeed, these two circadian systems may interact with each other. For example, the phase of the SCN may affect the phase of the FEO and vice versa. However, the coupling effect of the FEO on the SCN appears to be weaker than the coupling effect of the SCN on the FEO (Stephan, 1986; Ottenweller, Tapp, \& Natelson, 1990).

Recently, Ángeles-Castellanos, Amaya, SalgadoDelgado, Buijs, \& Escobar (2011) reported results similar to the present study. They showed that scheduled restricted feeding either before or after the LD advance accelerated the reentrainment of activity and body temperature rhythms. They also tested the effect of melatonin injection, which is known to accelerate the reentrainment of activity rhythms (Hau \& Gwinner, 1995; Marumoto, Murakami, Kuroda, \& Murakami, 1996) and found that the phase-shifting effects of scheduled feeding were even more pronounced than the effect of melatonin.

In conclusion, the present results show that scheduled feeding effectively accelerates the reentrainment of the motor activity rhythm in response to an LD advance. Together with the results obtained by ÁngelesCastellanos et al. (2011), the present study indicates that reentrainment of an SCN-controlled behavioral rhythm can be modulated by scheduled feeding under conditions that lead to uncoupling within the SCN (i.e., an LD shift). Nevertheless, further studies are necessary to evaluate how scheduled restricted feeding affects SCN activity during the process of reentrainment to new LD cycles. Finally, the results indicate that scheduled food intake may help in the readjustment process of circadian rhythms in humans who travel between time zones and ameliorate circadian rhythm disruptions.

\section{Acknowledgements}

The present work was supported by the Conselho Nacional de Desenvolvimento Científico e Tecnológico (CNPq, Brasil) and Fundação de Apoio à Pesquisa do Estado do Rio Grande do Norte (FAPERN, Brasil).

\section{References}

Agostino, P. V., Plano, S. A., \& Golombek, D. A. (2007). Sildenafil accelerates reentrainment of circadian rhythms after advancing light schedules. Proceedings of the National Academy of Sciences 
of the United States of America, 104, 9834-9839.

Ángeles-Castellanos, M., Amaya, J. M., Salgado-Delgado, R., Buijs, R. M., \& Escobar, C. (2011). Scheduled food hastens re-entrainment more than melatonin does after a 6 -h phase advance of the light-dark cycle in rats. Journal of Biological Rhythms, 26, 324-334.

Caldelas, I., Feillet, C. A., Dardente, H., Eclancher, F., Malan, A., (2005). Timed hypocaloric feeding and melatonin synchronize the suprachiasmatic clockwork in rats, but with opposite timing of behavioral output. European Journal of Neuroscience, 22, 921-929.

Campos, T. F., Silva, S. B., Fernandes, M. G., Araujo, J. F., \& Menezes, A. A. L. (2001). Diurnal variation in a visual-motor coordination test in healthy humans. Biological Rhythm Research, 32, 255-262.

Carneiro, B. T. S., \& Araujo, J. F. (2009). The food-entrainable oscillator: A network of interconnected brain structures entrained by humoral signals? Chronobiology International, 26, 1273-1289.

Coleman, G. J., \& Francis, A. J. (1991). Food deprivation and reinstatement phase shifts rat activity rhythms in constant light but not constant dark. Physiology and Behavior, 50, 167-171.

Costa, M. S. M. O., Santee, U. R., Cavalcante, J. S., Moraes, P. R. A., Santos, N. P., \& Britto, L. R. G. (1999). Retinohypothalamic projections in the common marmoset (Callithrix jacchus): A study using cholera toxin subunit B. Journal of Comparative Neurology, 415, 393-403.

Daan, S., \& Pittendrigh, C. S. (1976). A functional analysis of circadian pacemakers in nocturnal rodents: III. Heavy water and constant light: homeostasis of frequency? Journal of Comparative Physiology: A. Neuroethology, Sensory, Neural, and Behavioral Physiology, 106, 267-290.

Dibner, C., Schibler, U., \& Albrecht, U. (2010). The mammalian circadian timing system: Organization and coordination of central and peripheral clocks. Annual Review of Physiology, 72, 517-549.

Guan, X., Hess, J. F., Yu, H., Hey, P. J., \& van der Ploeg, L. H. T. (1997) Differential expression of $m R N A$ for leptin receptors isoforms in the rat brain. Molecular and Cellular Endocrinology, 133, 1-7.

Hara, R., Wan, K., Wakamatsu, H., Aida, R., Moriya, T., Akiyama, M., \& Shibata, S. (2001). Restricted feeding entrains liver clock without participation of the suprachiasmatic nucleus. Genes to Cells, 6, 269-278.

Hau, M., \& Gwinner, E. (1995). Continuous melatonin administration accelerates resynchronization following phase shifts of a lightdark cycle. Physiology and Behavior, 58, 89-95.

Johnson, R. F., Morin, L. P., \& Moore, R. Y.(1988). Retinohypothalamic projections in the hamster and rat demonstrated using cholera toxin. Brain Research, 462, 301-312.

Kondo, T., Strayer, C. A., Kulkarni, R. D., Taylor, W., Ishiura, M., Golden, S. S., \& Johnson, C. H. (1993). Circadian rhythms in prokaryotes: luciferase as a reporter of circadian gene expression in cyanobacteria. Proceedings of the National Academy of Sciences of the United States of America, 90, 5672-5676.

Lamont, E. W., Diaz, L. R., Barry-Shaw, J., Stewart, J., \& Amir, S. (2005). Daily restricted feeding rescues a rhythm of period2 expression in the arrhythmic suprachiasmatic nucleus. Neuroscience, 132, 245-248.

Marchant, E. G., \& Mistlberger, R. E. (1997). Anticipation and entrainment to feeding time in intact and SCN-ablated C57BL/6j mice. Brain Research, 765, 273-282.

Marumoto, N., Murakami, N., Kuroda, H., \& Murakami, T. (1996). Melatonin accelerates reentrainment of circadian locomotor activity rhythms to new light-dark cycles in the rat. Japanese Journal of Physiology, 46, 347-351.

Mendoza, J., Graff, C., Dardente, H., Pevet, P., \& Challet, E. (2005). Feeding cues alter clock gene oscillations and photic responses in the suprachiasmatic nuclei of mice exposed to a light/dark cycle Journal of Neuroscience, 25, 1514-1522.
Mistlberger, R. E. (1993). Effects of scheduled food and water access on circadian rhythms of hamsters in constant light, dark, and light:dark. Physiology and Behavior, 53, 509-516.

Mistlberger, R. E. (1994). Circadian food-anticipatory activity: formal models and physiological mechanisms. Neuroscience and Biobehavioral Reviews, 18, 171-195.

Mistlberger, R. E. (2009). Food-anticipatory circadian rhythms: Concepts and methods. European Journal of Neuroscience, 30, 1718-1729.

Mistlberger, R. E., Sinclair, S. V., Marchant, E. G., \& Neil, L. (1997). Phase shifts to refeeding in the Syrian hamster mediated by running activity. Physiology and Behavior, 61, 273-278.

Mrosovsky, N., \& Salmon, P. A. (1987). A behavioural method for accelerating re-entrainment of rhythms to new light-dark cycles. Nature, 330, 372-373.

Nagano, M., Adachi, A., Nakahama, K., Nakamura, T., Tamada, M., (2003). An abrupt shift in the day/night cycle causes desynchrony in the mammalian circadian center. Journal of Neuroscience, 23, 6141-6151.

Nascimento, E. S., Jr., Souza, A. P. M., Duarte, R. B., Magalhães, M. A. F., Silva, S. F.,(2010). The suprachiasmatic nucleus and the intergeniculate leaflet in the rock cavy (Kerodon rupestris): retinal projections and immunohistochemical characterization. Brain Research, 1320, 34-46.

Ohta, H., Yamazaki, S., \& McMahon, D. G. (2005). Constant light desynchronizes mammalian clock neurons. Nature Neuroscience, $8,267-269$

Ottenweller, J. E., Tapp, W. N., \& Natelson, B. H. (1990). Phaseshifting the light-dark cycle resets the food-entrainable circadian pacemaker. American Journal of Physiology: Regulatory, Integrative and Comparative Physiology, 258, R994-R1000.

Prosser, R. A., \& Bergeron H. E. (2003). Leptin phase-advances the rat suprachiasmatic circadian clock in vitro. Neuroscience Letters $336,139-142$.

Simonetta, S. H., Migliori, M. L., Romanowski, A., \& Golombek, D. A. (2009). Timing of locomotor activity circadian rhythms in Caenorhabidits elegans. PloS One, 4, e7571.

Simpson, K. A., Martin, N. M., \& Bloom, S. R. (2009). Hypothalamic regulation of food intake and clinical therapeutic applications. Arquivos Brasileiros de Endocrinologia \& Metabologia, 53, 120-128.

Stephan, F. K., \& Zucker, I. (1972). Circadian rhythms in drinking behavior and locomotor activity of rats are eliminated by hypothalamic lesions. Proceedings of the National Academy of Sciences of the United States of America, 69, 1583-1586.

Stephan, F. K. (1986). Coupling between feeding- and lightentrainable circadian pacemakers in the rat. Physiology and Behavior, 38, 537-544.

Stephan, F. K., Swann, J. M., \& Sisk, C. L. (1979). Entrainment of circadian rhythms by feeding schedules in rats with suprachiasmatic lesions. Behavioral and Neural Biology, 25, 545-554.

Stokkan, K., Yamazaki, S., Tei, H., Sakaki, Y., \& Menaker, M. (2001) Entrainment of the circadian clock in the liver by feeding. Science, 291, 490-493.

Waterhouse, J., Reilly, T., \& Atkinson, G. (1997). Jet-lag. Lancet, 350, 1611-1616.

Yannielli, P. C., Molyneux, P. C., Harrington, M. E., \& Golombek, D. A. (2007). Ghrelin effects on the circadian system of mice. Journal of Neuroscience, 27, 2890-2895.

Yi, C., van der Vliet, J., Dai, J., Yin, G., Ru, L., \& Buijs, R. M. (2006). Ventromedial arcuate nucleus communicates peripheral metabolic information to the suprachiasmatic nucleus. Endocrinology, 147, 283-294.

Zigman, J. M., Jones, J. E., Lee, C. E., Saper, C. B., \& Elmquist, J. K. (2006). Expression of ghrelin receptor mRNA in the rat and the mouse brain. Journal of Comparative Neurology, 494, 528-548. 